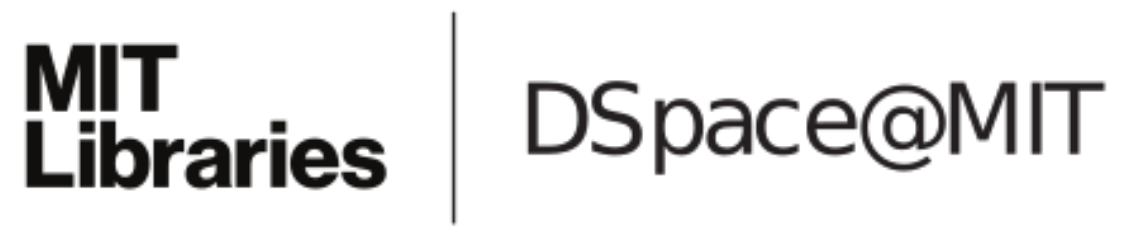

\author{
MIT Open Access Articles
}

Enhancement of thermoelectric figure-of-merit by resonant states of aluminium doping in lead selenide

The MIT Faculty has made this article openly available. Please share how this access benefits you. Your story matters.

Citation: Zhang, Qinyong, Hui Wang, Weishu Liu, Hengzhi Wang, Bo Yu, Qian Zhang, Zhiting Tian, et al. "Enhancement of Thermoelectric Figure-of-Merit by Resonant States of Aluminium Doping in Lead Selenide." Energy \& Environmental Science 5, no. 1 (2012): 5246.

As Published: http://dx.doi.org/10.1039/c1ee02465e

Publisher: Royal Society of Chemistry

Persistent URL: http://hdl.handle.net/1721.1/86905

Version: Author's final manuscript: final author's manuscript post peer review, without publisher's formatting or copy editing

Terms of use: Creative Commons Attribution-Noncommercial-Share Alike 


\title{
Enhancement of Thermoelectric Figure-of-Merit by Resonant States of Aluminum Doping in Lead Selenide
}

\author{
Qinyong Zhang ${ }^{a, b}$, Hui Wang ${ }^{b}$, Weishu Liu $^{b}$, Bo Yu ${ }^{b}$, Qian Zhang ${ }^{b}$, Hengzhi Wang ${ }^{b}$, George \\ $\mathrm{Ni}^{c}$,Sangyeop Lee ${ }^{c}$, Zhiting Tan $^{c}$, Keivan Esfarjani ${ }^{c}$, Gang Chen*c, and Zhifeng Ren*b \\ ${ }^{a}$ School of Material Science and Engineering, Xihua University, Chengdu, Sichuan 610039, P. R. China \\ ${ }^{b}$ Department of Physics, Boston College, Chestnut Hill, Massachusetts 02467, USA. Fax: 1-617-552-8478; Tel: 1-617-552-2832; E-mail: \\ renzh@bc.edu \\ ${ }^{c}$ Department of Mechanical Engineering, Massachusetts Institute of Technology, Cambridge, Massachusetts 02139, USA. Fax: 1-617-324-5519; \\ Tel: 1-617-253-0006; E-mail: gchen2@mit.edu
}

By adding aluminium (Al) into lead selenide (PbSe), we successfully prepared n-type PbSe thermoelectric materials with a figure-of-merit $(Z T)$ of 1.3 at $850 \mathrm{~K}$. Such high $Z T$ is achieved by a combination of high Seebeck coefficient caused by very possibly the resonant states in the conduction band created by Al dopant and low thermal conductivity from nanosized phonon scattering centers.

\section{Broader context}

Thermoelectric devices directly convert heat to electricity, thus they are important for harvesting natural heat as well as waste heat. For efficient devices, high figure-of-merit $(Z T)$ materials are desired. We report here n-type lead selenide (PbSe) thermoelectric materials with $Z T$ of 1.3 at $850 \mathrm{~K}$. These materials are prepared by adding aluminium (Al) in $\mathrm{PbSe}$ during ball milling and hot pressing. $\mathrm{Al}$, as a dopant in $\mathrm{PbSe}$, possibly creates resonant states in the conduction band and causes increase in the local density of states (DOS) near the Fermi level. As a result, the Seebeck coefficients of Al added PbSe samples are about $40 \sim 100 \%$ higher than the predicted values by the simple parabolic band model and about $40 \%$ higher than the Cl-doped reference $\mathrm{PbSe}$ sample without resonant states. Furthermore, using ball milling and hot pressing technique, the structure of our samples contains features such as $\mathrm{Pb}$ depleted discs, small grains, and $\sim 10 \mathrm{~nm}$ subgrains that are effective for phonon scattering, and result in a much lower lattice thermal conductivity of $0.6 \sim 0.7 \mathrm{Wm}^{-1} \mathrm{~K}^{-1}$ at $850 \mathrm{~K}$. Further improvement could be expected by even better control of the Fermi level through optimization of addition of other doping materials.

Thermoelectric (TE) materials and devices have drawn significant interests and attentions for the past decades due to their potential applications in converting solar energy and waste heat into electricity ${ }^{1}$. The performance of thermoelectric materials depends on dimensionless figure-of-merit, $Z T=S^{2} \sigma T /\left(\kappa_{\text {lat }}+\kappa_{\text {carr }}\right)$, where $S, \sigma, T, \kappa_{\text {lat }}$, and $\kappa_{\text {carr }}$ are Seebeck coefficient, electrical conductivity, absolute temperature, lattice thermal conductivity, and electronic thermal conductivity, respectively ${ }^{2}$. Improvements on $Z T$ can be achieved by either increasing the power factor $\left(S^{2} \sigma\right)$ or reducing the thermal conductivity, or even better, by simultaneously increasing the power factor and reducing the thermal conductivity ${ }^{3}$. Potential ways to improve the power factor are through increasing the Seebeck coefficient $(S)$ via band modification ${ }^{4}$ or engineering ${ }^{5}$, energy barrier filtering ${ }^{6-8}$, additional carrier pocket ${ }^{9}$, and resonant $\operatorname{states}^{10}$, or enhancing the mobility such as modulation doping ${ }^{11}$. Among these possibilities, resonant states and additional carrier pockets have led to net increased $Z T$ values via the power factor enhancements 9,10 . In contrast, many methods have been found to be effective in reducing lattice thermal conductivity, for example, alloy scattering, boundary scattering, nanocomposites or nanoinclusion routes, etc. Simultaneously achieving resonant states to enhance the Seebeck coefficient thus the power factor and nanoscale grain boundary scattering to reduce the lattice thermal conductivity have not been reported before.

As one of the most studied bulk TE materials, PbTe based materials get a significantly enhancement recently by introducing various nano inclusions or modifying the band structure, and reach a high $Z T$ value of 1.5 1.8, such as p-type $\mathrm{PbTe} \mathrm{H}_{1-\mathrm{x}} \mathrm{Se}_{\mathrm{x}}: \mathrm{Na}$ $(\sim 1.8)^{5}$, PbTe-Sr $\mathrm{Pr}_{2} \mathrm{Na}(\sim 1.7)^{12}$, PbTe:Tl $(\sim 1.5)^{4,6}, \mathrm{NaPb}_{\mathrm{m}} \mathrm{SbTe}_{2+\mathrm{m}}(\sim 1.7)^{13}$, and n-type AgPb $\mathrm{SbTe}_{2+\mathrm{m}}(\sim 1.7)^{14}$ and PbTe$\mathrm{PbS}: \mathrm{PbI}_{2}(\sim 1.5)^{15}$. All these materials are based on Te. However, Te is extremely rare in the earth crust, just $0.001 \mathrm{ppm}$, which is even less than $0.004 \mathrm{ppm}$ for $\mathrm{Au}$ and $0.005 \mathrm{ppm}$ for Pt This will limit the wide applications of PbTe-based materials. To develop materials without $\mathrm{Te}$ or less Te will be very urgent and important. Although $\mathrm{PbS}$ and $\mathrm{PbSe}$ are very similar to $\mathrm{PbTe}$ in crystal structure, band structure, and many other properties ${ }^{17}$, they are much less studied than PbTe because of the concerns that the smaller size of S or Se and lighter atomic mass in comparison with Te potentially result in higher lattice thermal conductivity.

Recently, a theoretical calculation of PbSe by Parker et al. ${ }^{16}$ showed that due to the flat feature of energy diagram in valence band, the p-type PbSe may possibly reach $Z T \sim 2$ at $1000 \mathrm{~K}$ if it is heavily doped. Based on this suggestion, Wang et al. doped $\mathrm{Na}$ into $\mathrm{PbSe}^{17}$, and got a peak $Z T$ value of around 1.2 at $850 \mathrm{~K}$, which is $15 \%$ lower than 1.4 of Na doped PbTe. Comparing to ptype, the highest $Z T$ of n-type PbSe was limited to $\sim 0.9^{18}$, even in the recently reported $\mathrm{Ga}$ and In doped $\mathrm{PbSe}^{19}$. 
In this paper, we present the thermoelectric properties of the unexpected n-type PbSe samples by addition of Al prepared by ball milling and hot pressing. The highest $Z T \mathrm{~s}$ we achieved are $\sim 1.3$ due to the combined effects of high Seebeck coefficient and low thermal conductivity, which is comparable to 1.4 of n-type Iodine doped PbTe with no special nano inclusions ${ }^{20}$. Intuitively, $\mathrm{Al}$ with less valence electron than $\mathrm{Pb}$, it should be acceptor when it substitutes $\mathrm{Pb}$. However, our experimental results show that $\mathrm{Al}$ addition to PbSe make it n-type unexpectedly. To our knowledge, this is the first report regarding the effect of $\mathrm{Al}$ doping on thermoelectric properties of lead chalcogenides, and also the first observation on enhancement of Seebeck coefficient by creating resonant states in n-type $\mathrm{PbSe}$.

Element $\mathrm{Pb}$ (granules, 99.99\%), Se (granules, 99.999\%), and Al (powder, 99.999\%) from Alfa Aesar were weighed according to the stoichiometry of $\mathrm{PbSe}: \mathrm{Al}_{\mathrm{x}}(\mathrm{x}=0,0.005,0.01,0.02$, and 0.03 , with higher concentration also experimented but not presented here due to worse properties $), \mathrm{PbSe}_{\mathrm{Cl}}(\mathrm{x}=0.002,0.01), \mathrm{PbSe}: \mathrm{I}_{\mathrm{x}}(\mathrm{x}=0.005)$ and loaded in a stainless jar with stainless balls for mechanical alloying by a high energy ball mill SPEX 8000D (SPEX SamplePrep). The alloyed nanopowders were then loaded into a graphite die with an inner diameter of $12.7 \mathrm{~mm}$, and finally consolidated by direct current (DC) induced hot pressing $\mathrm{s}^{3,21,22}$.

The thermal diffusivities of the as-pressed disk samples were measured by a laser flash system (NETZSCHLFA 457), and the specific heat was determined by a DSC system (NETZSCH DSC 200-F3). Thermal conductivity was then calculated as the product of the thermal diffusivity, specific heat, and volumetric density that was determined by the Archimedes' Method. Bar samples with dimensions of about $2 \times 2 \times 12 \mathrm{~mm}$ were cut from the disk for measuring the Seebeck coefficient and electrical conductivity simultaneously by a four-point probe system (ULVAC ZEM-3). Thin disk samples of around $0.5 \mathrm{~mm}$ in thickness were prepared for Hall measurements on a Lakeshore system (Hall Effect System7712A). The structures of the as-pressed samples were studied by X-ray diffraction (Rigaku RU300) using $\mathrm{Cr}$ radiation $\left(\mathrm{K}_{\alpha}: 2.8976 \AA\right.$ ), field emission scanning electron microscopy (SEM, JEOL-6340F), and transmission electron microscopy (TEM, JEOL-2010F).

A first principles calculation was conducted using the PWscf code to investigate the effect of $\mathrm{Al}$ on PbSe. In our calculation, fully relativistic pseudo-potentials are used. We first construct a $2 \times 2 \times 2 \mathrm{PbSe}$ supercell with 64 atoms, then we put 1 Al atom into the system $(\sim 1.5 \%)$ either substituting $\mathrm{Pb}$ or Se or sitting at the interstitial place. We relax the systems separately and perform the calculation on the relaxed systems, namely $\mathrm{Al}$ replaces $\mathrm{Pb}\left(\mathrm{AlPb}_{31} \mathrm{Se}_{32}\right)$ or $\mathrm{Al}$ replaces $\mathrm{Se}\left(\mathrm{Pb}_{32} \mathrm{Se}_{31} \mathrm{Al}\right)$ or $\mathrm{Al}$ goes to interstitial site $\left(\mathrm{AlPb}_{32} \mathrm{Se}_{32}\right)$. The formation energy was also calculated to find out the most favourable location for $\mathrm{Al}$ among all the three possible cases.

Dense samples (at least $96.3 \%$ of the theoretical density) of $\mathrm{PbSe}: \mathrm{Al}_{\mathrm{x}}$ with different $\mathrm{Al}$ doping concentrations $(\mathrm{x}=0,0.005$, $0.01,0.02,0.03$, and 0.04 ) were prepared, and their X-ray diffraction patterns are shown in Fig. 1, which indicate that all the samples are crystallized in rock salt structure. In the range of $x=0 \sim 0.03$, no visible impurity phase could be detected. Impurity phase in samples with $\mathrm{x}$ higher than 0.03 were indeed detected, resulting in worse thermoelectric properties. The lattice parameters of all the samples were calculated by using Rietveld refinement, which was performed using an X'Pert HighScore Plus software (PANalytical, X'Pert Pro). The lattice parameter $a$ of all the samples is 6.130-6.133 $\AA$ with error bar of $0.002 \AA$. No clear doping concentration dependent increase or decrease in lattice parameter was observed.

Figure $2 \mathrm{a}$ plot the room temperature Seebeck coefficient of $\mathrm{Al}$ doped $\mathrm{PbSe}$ as a function of carrier concentration. It is noted that the Seebeck coefficients of our $\mathrm{PbSe}_{\mathrm{Al}} \mathrm{l}_{\mathrm{x}}(\mathrm{x}=0 \sim 0.03)$ samples are all negative showing the nature of $\mathrm{n}$-type conduction. It was reported that Indium could work as n-type dopant in $\mathrm{PbSe}{ }^{19,23}$. However, the reason is still unknown. We guess Al and In may get in to the interstitial site, similar with $\mathrm{Cu}$ in $\mathrm{Bi}_{2} \mathrm{Te}_{2.7} \mathrm{Se}_{0.3}$, acting as n-type dopant ${ }^{24}$. However, we did not observe any lattice parameter increase in $\mathrm{Al}$ doped $\mathrm{PbSe}$, meaning ineterstitial case is not correct. So substitution of $\mathrm{Al}$ for $\mathrm{Pb}$ or $\mathrm{Te}$ may be the case. A theoretical calculation clearly showed that $\mathrm{Al}$ replacing $\mathrm{Pb}$ is the reasonable result and causing both $\mathrm{n}$-type conduction and resonant states. 


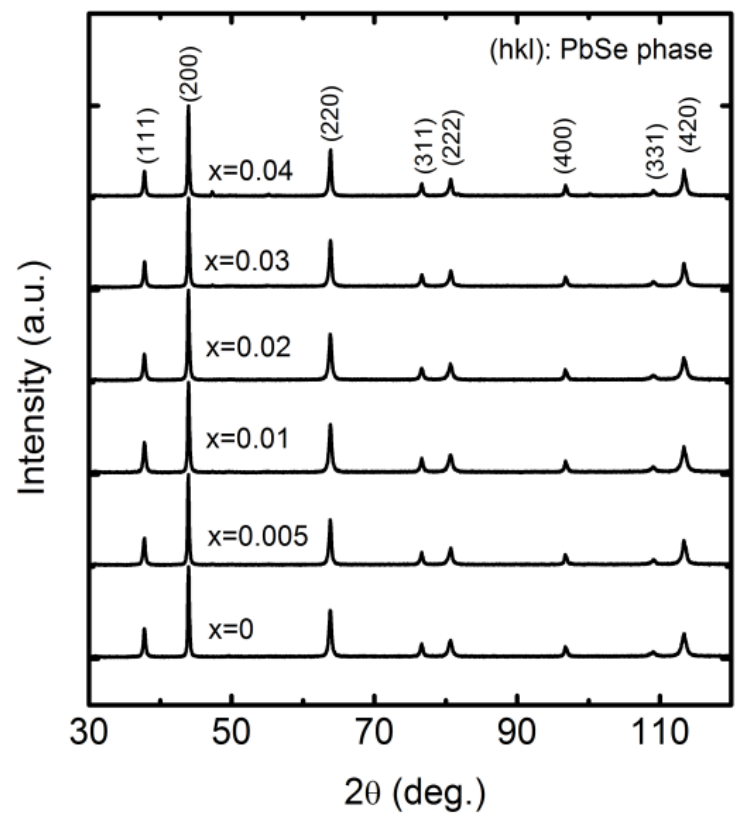

Fig. 1 XRD spectra of PbSe:Al samples with different Al concentrations of $\mathrm{x}=0,0.005,0.01,0.02,0.03$, and 0.04 .

The theoretical Pisarenko curves for both n- and p-type materials were calculated using a simple parabolic band model $(\mathrm{SPB})^{25}$, as shown in Eqs. (1) and (2). We choose acoustic phonon scattering as the dominance electron scattering mechanism (s $=-1 / 2)$, and the effective mass of $\mathrm{m} * / \mathrm{m}_{0}=0.28$ according to $\mathrm{PbSe}: \mathrm{Na}^{17}$.

$$
\begin{gathered}
S= \pm \frac{k_{B}}{e}\left(\frac{(5 / 2+r) F_{3 / 2+r}(\eta)}{(3 / 2+r) F_{1 / 2+r}(\eta)}-\eta\right) \\
n_{H}=4 \pi\left(\frac{2 m^{*} k_{B} T}{h^{2}}\right)^{3 / 2} \frac{F_{1 / 2}(\eta)}{R_{H}}
\end{gathered}
$$

In Eqs. (1) and (2), $S, k_{B}, e$, and $r$ are Seebeck coefficient, Boltzmann constant, electron charge, and scattering parameter, respectively. The $F_{i}(\eta)$ is the Fermi integral and $\eta$ is the reduced Fermi energy. In Fig. 2a, Seebeck coefficient of the pure PbSe sample (no Al added with a carrier concentration of $\left.\sim 3.1 \times 10^{18} \mathrm{~cm}^{-3}\right)$, Cl-doped PbSe samples $(0.2 \%$ and $1 \% \mathrm{Cl})$, I doped $\mathrm{PbSe}$ sample $(0.5 \% \mathrm{I})$ lies right on the n-type Pisarenko curve, indicating good agreement with the expectation of SPB model. The reference samples with $\mathrm{Cl}^{18,23}$ for n-type, Na17 for p-type also fit the Pisarenko curve. In other words, there is no evidence of enhancement of Seebeck coefficient by possible resonant states in normal n-type PbSe with $\mathrm{Cl}$ or I dopants. However, the situation for $\mathrm{Al}$ in $\mathrm{PbSe}$ is totally different: when the carrier concentration increases to $1.78 \sim 3.96 \times 10^{19} \mathrm{~cm}^{-3}$ upon 0.5 to 3 at.\% $\mathrm{Al}$ doping, the Seebeck coefficient almost remains constant at around $-111 \mu \mathrm{VK}^{-1}$, about $40 \sim 100 \%$ higher than that from the SPB model and experimental values of $\mathrm{PbSe}: \mathrm{Cl}$ samples with the same carrier concentration. Similar observation was also found in $\mathrm{p}$ type $\mathrm{PbTe}$ :Tl samples ${ }^{4}$, which was attributed to the increase of the local DOS near Fermi level by presence of resonant states. The resonant state is an additional carrier pocket close to the major carrier band ${ }^{9}$, which is believed to increase to the $S^{2} n$ and hence the power factor. 

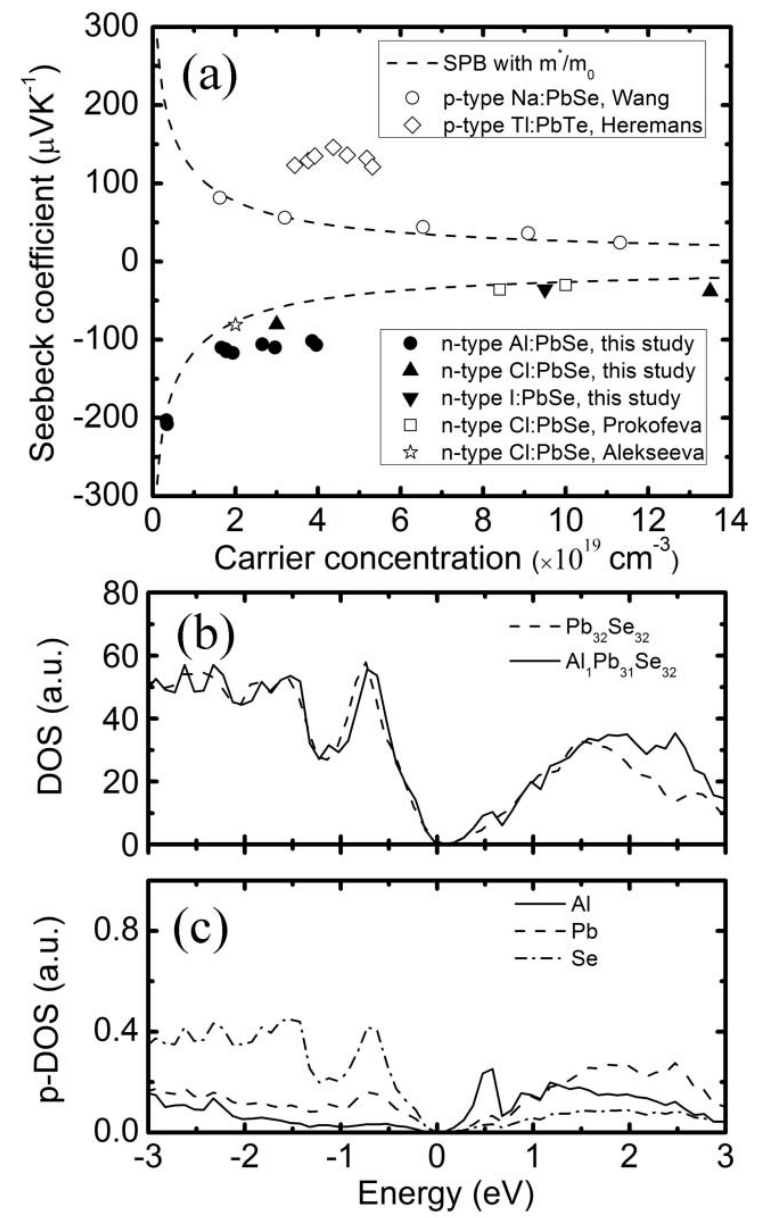

Fig. 2 (a) Dependence of Seebeck coefficient on carrier concentration at RT. Solid lines are theoretical Pisarenko curve with $\mathrm{m}^{*} / \mathrm{m}_{0}=0.28$. Filled circles and partially filled triangles represent results of this study. (b) local DOS of $\mathrm{Pb}_{32} \mathrm{Se}_{32}$ and $\mathrm{AlPb}_{31} \mathrm{Se}_{32}$; (c) local $\mathrm{p}-\mathrm{DOS}$ for each atom: $\mathrm{Al}$, $\mathrm{Pb}$, Se density of state of $\mathrm{AlPb}_{31} \mathrm{Se}_{32}$. A resonant sate was shown near the conduction band edge of PbSe caused by Al. The valence band top was chosen as the zero energy in (b) and (c).

In order to find out which lattice site $(\mathrm{Pb}, \mathrm{Se}$, interstitial) in $\mathrm{PbSe}$ is more favourable for $\mathrm{Al}$ atom and why the Seebeck coefficient remains the same with increased carrier concentration, we conducted first-principle calculations based on density function theory with $2 \times 2 \times 2$ supper cell. Firstly, the formation energy of two most possible cases, Al substituting $\mathrm{Pb}$ and the interstitial site, was calculated. The formation energy for the substitution case $\left(\mathrm{AlPb}_{31} \mathrm{Se}_{32}\right)$ is defined as $E_{\text {sub }}=E\left(\mathrm{AlPb}_{31} \mathrm{Se} \mathrm{C}_{32}\right)$ $E\left(\mathrm{~Pb}_{31} \mathrm{Se}_{32}\right)-E(\mathrm{Al})$, while for the interstitial case, it is $E_{\text {int }}=E\left(\mathrm{AlPb}_{32} \mathrm{Se}_{32}\right)-E\left(\mathrm{~Pb}_{32} \mathrm{Se}_{32}\right)-E(\mathrm{Al})$. Finally, we get the formation energy of substitution case $\left(E_{s u b}=-0.45 \mathrm{Ry}\right)$, lower than that of the interstitial case $\left(E_{\text {int }}=-0.18 \mathrm{Ry}\right)$, which means that the substitution case is more energy favourable. Based on the band structure calculation, the density of state (DOS) for both substitution and interstitial case was obtained. A small bump near the conduction band is found in the substitution case as compared with the undoping case as shown in Fig. 2b. A partial DOS for each atom including $\mathrm{Al}, \mathrm{Pb}$, and Se is shown in Fig. 2c. A similar resonant state was also observed in $\mathrm{Al}$ doped $\mathrm{Pb}_{1-\mathrm{x}} \mathrm{Al}_{\mathrm{x}} \mathrm{Te}^{26,27}$. It is clearly suggested that a resonant state in the conduction band is caused by the $1.5 \% \mathrm{Al}$ in $\mathrm{Pb}_{1-\mathrm{x}} \mathrm{Al}_{\mathrm{x}} \mathrm{Se}$. However, we did not observe similar resonant state near the band edge in the interstitial case (i.e., $\mathrm{AlPb}_{32} \mathrm{Se}_{32}$ ) according to our first principle calculation. Combining the experimental and theoretical results, we come to a conclusion that $\mathrm{Al}$ likely replaces $\mathrm{Pb}$ rather than sits at the interstitial site. 


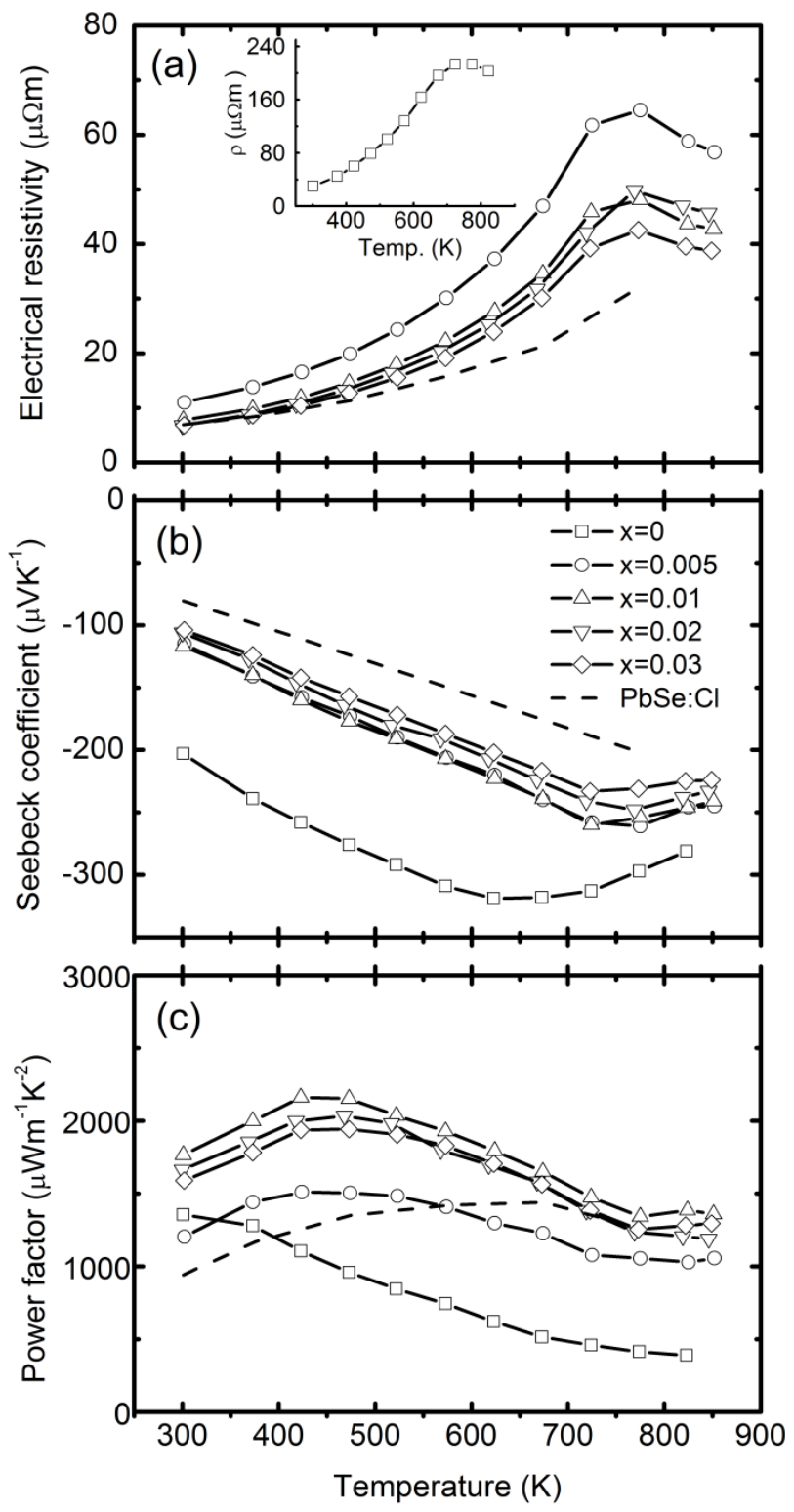

Fig. 3 Temperature dependent thermoelectric properties of $\mathrm{PbSe}: \mathrm{Al}_{\mathrm{x}}(\mathrm{x}=0,0.005,0.01,0.02$, and 0.03$)(\mathrm{a})$ electrical resistivity, (b) Seebeck coefficient, and (c) power factor. The inset of (a) is the electrical resistivity of pure PbSe sample without Al dopant. The dash line is for the $0.2 \%$ $\mathrm{Cl}$-doped PbSe.

It is reported that the modification of band edge also can be realized by nano inclusion ${ }^{28,29}$, such as the $\mathrm{AgSbTe}_{2}$ nanoinclusion in the $\mathrm{PbTe}$ matrix ${ }^{30}$. Since such nano inclusions are widely observed in nanocomposite made by ball milling and hot pressing ${ }^{3,21,22}$, the Al related nano inclusion may be another possible reason for the unchanged Seebeck coefficient increasing carrier concentration. Besides the possible band structure changing by resonant state or nano inclusion, there is another possibility, i.e. an increase in scattering parameter $r$. This possible reason for the enhanced Seebeck coefficient can be interpreted from the Mott expression of Seebeck coefficient which is shown as following,

$$
S=\frac{\pi^{2}}{3} \frac{k_{B}}{e} k_{B} T\left\{\frac{1}{n} \frac{d n(E)}{d E}+\frac{1}{\mu} \frac{d \mu(E)}{d E}\right\}_{E=E_{F}}
$$


where $\mathrm{n}, \mu, \mathrm{E}$, and $\mathrm{E}_{\mathrm{F}}$ are carrier concentration, mobility, energy of carriers, and Fermi level, respectively. The increase in $\frac{d \mu(E)}{d E}$ is equivalent to an increased scattering parameter $r$. The changing in $r$ can be achieved from the resonant scattering which involves electron jumping in and out between the normal band and the quasi-local impurity state. Unfortunately, we do not have enough evidence for the existence of such resonant scattering in our Al doped PbSe samples.

The detailed thermoelectric properties of PbSe:Al samples are presented in Fig. 3 together with the data of $0.2 \% \mathrm{Cl}$-doped $\mathrm{PbSe}$ sample for comparison. The Cl-doped $\mathrm{PbSe}$ is made by using the same method. It is found that $\mathrm{Al}$ is a much weaker $\mathrm{n}$-type dopant than $\mathrm{Cl}$. The carrier concentration of $0.2 \% \mathrm{Cl}$-doped $\mathrm{PbSe}\left(3.0 \times 10^{19} \mathrm{~cm}^{-3}\right)$ is comparable to that of $2 \% \mathrm{Al}-\mathrm{doped} \mathrm{PbSe}$ $\left(2.7-2.9 \times 10^{19} \mathrm{~cm}^{-3}\right)$. However, the Seebeck coefficient $\left(-80 \mu \mathrm{VK}^{-1}\right)$ of the $0.2 \% \mathrm{Cl}$-doped PbSe is much lower than that $(-106$ $\mu \mathrm{VK}^{-1}$ ) of the $2 \% \mathrm{Al}$-doped $\mathrm{PbSe}$, which demonstrates that $\mathrm{PbSe}: \mathrm{Al}$ has larger effective mass than the $0.2 \% \mathrm{Cl}-\mathrm{doped} \mathrm{PbSe}$. This fact could be a direct evidence for the existence of resonant state. Owing to the enhanced Seebeck coefficient, a significantly higher power factor of $2030 \mu \mathrm{Wm}^{-1} \mathrm{~K}^{-2}$ was obtained in the $2 \% \mathrm{Al}$-doped $\mathrm{PbSe}$ compared with $1440 \mu \mathrm{Wm}^{-1} \mathrm{~K}^{-2}$ for the $0.2 \% \mathrm{Cl}$ doped $\mathrm{PbSe}$.

Total thermal conductivities of the studied samples, demonstrated in Fig. 4, were calculated using $\kappa=\rho D C_{p}$, where $\rho$ is volumetric density, $D$ the diffusivity (Fig. $4 \mathrm{a}$ ), and $C_{p}$ the specific heat (Fig. $4 \mathrm{a}$ ). In calculation of $\kappa$, the $C_{p}$ values of x=0.01 are used for $\mathrm{x}=0.005,0.01$ samples, and that of $\mathrm{x}=0.03$ are used for $\mathrm{x}=0.02,0.03$ samples. The thermal conductivity of $\mathrm{PbSe}: \mathrm{Al}$ samples are $\sim 0.9 \mathrm{Wm}^{-1} \mathrm{~K}^{-1}$ at $850 \mathrm{~K}$. The lattice thermal conductivity is calculated by subtracting the electronic contribution from the total thermal conductivity ( $\kappa_{\text {lat }}=\kappa-\kappa_{\text {carr }}=\kappa-L \sigma T$, where $L$ is the Lorenz number). Here, the Fermi energy related Lorenz number is calculated by using the Fermi energy extracted from Seebeck coefficient within a simple parabolic band model, and the acoustic phonon scattering as the major carrier scattering mechanism ${ }^{31}$. As a result, the Lorenz number at room temperature is ranged from $1.61 \times 10^{-8}$ to $1.87 \times 10^{-8} \mathrm{~V}^{2} \mathrm{~K}^{-2}$ depending on the $\mathrm{Al}$ concentartion. The $\kappa_{\text {lat }}$ of pure PbSe sample at $\mathrm{RT}$ is $1.81 \mathrm{Wm}{ }^{-}$ ${ }^{1} \mathrm{~K}^{-1}$, consistent with the reported data ${ }^{17}$. The $\kappa_{\text {lat }}$ of Al-doped samples is lower and decreases with Al concentration. It is very interesting to note that the Al-doped samples have $\kappa_{\text {lat }}$ as low as $0.58 \sim 0.69 \mathrm{Wm}^{-1} \mathrm{~K}^{-1}$ at $850 \mathrm{~K}$. This low $\kappa_{\text {lat }}$ is related to the microstructures discussed below. The highest $Z T$ of around 1.3 is achieved at $\mathrm{x}=0.01$ at $850 \mathrm{~K}$, which is $\sim 300 \%$ and $40 \%$ higher than that of the un-doped and Cl-doped samples, respectively. 


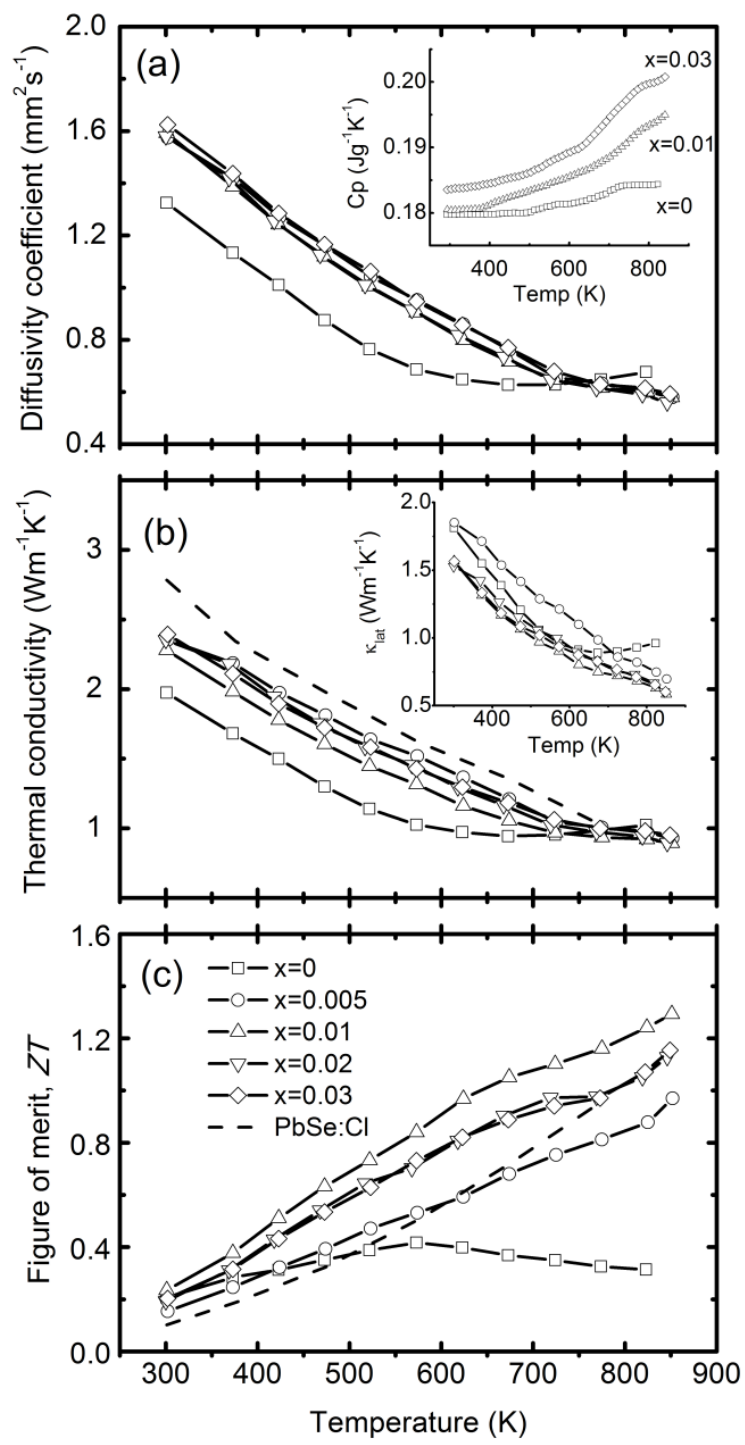

Fig. 4. Temperature dependent thermoelectric properties of $\mathrm{PbSe}: \mathrm{Al}_{\mathrm{x}}(\mathrm{x}=0,0.005,0.01,0.02$, and 0.03$)$, $\mathrm{x}$ values are wrong in the figures (a) diffusivity coefficient with the specific heat $C_{p}$ as the inset, (b) thermal conductivity with the lattice thermal conductivity as the inset, and (c) figure of merit.

In order to elucidate the reasons for such a low lattice thermal conductivity of PbSe:Al samples, extensive microscopy study was conducted by SEM and TEM. The following microscopic characteristics could be essential to theoretically explain the ultralow lattice thermal conductivity. Firstly, SEM microphotograph in Fig. 5a shows that the average grain size of PbSe:Al samples is less than $1 \mu \mathrm{m}$, which was analyzed by linear intercept particle analyzing method ${ }^{32}$, and confirmed by TEM bright field image shown in Fig. 5b. Secondly, Fig. 5a and 5b show that there are many nanosized pores (sphere-like, around $50 \mathrm{~nm}$ in diameter) distributing along the grain boundaries (mostly) and inside the grains (some), with the latter pores indicated by arrows in Fig. 5a and Fig. 5b. These small crystalline grains and the nanosized pores could mainly result in the reduced lattice thermal conductivity due to strong scattering to the phonons. Thirdly, it is interesting to observe that there are a lot of Pb-depleted disks lying on the $\{100\}$ planes in PbSe:Al grains with diameter around $2 \sim 5 \mathrm{~nm}$ and thickness less than $0.5 \mathrm{~nm}$, as shown in Fig. 5c. With a volumetric density of about $9 \times 10^{17} \mathrm{~cm}^{-3}$, the Pb-depleted disks in Fig. $5 \mathrm{c}$ may cause significant lattice strain fields (3-20 nm) on both sides of the disks along their normal directions (see the arrow directions). This kind of Pb-depleted disks has been reported in our previous work ${ }^{33}$ in $\mathrm{PbTe}$ system. Due to the lack of $\mathrm{Pb}$ atomic layers in the crystal, both the disks and the related lattice strain fields could also exert additional scattering effect on phonon transporting and lead to the further reduced lattice thermal conductivity. Fourthly, the atomic scale microphotographs in Fig. 5d and 5e clearly show the contrast differences related to atomic columns and even a tilt contrast column, as separately pointed out by the arrows in Fig. 5e. Although Fig. 5e is only a modified HRTEM lattice image rather than a structural image, it is suggested that these weaker white spots were caused by the Al doping. Accordingly, the atomically scaled inhomogeneity might affect the phonon scattering in each grain. Finally, along the 
[111] direction, Fig. 5f shows that there are many distinctive lattice areas, named subgrains (enclosed by white circles), in each grain. It is noted that these nanoscaled subgrains are separated by obscure boundary regions. It is suggested that these subgrain boundaries may come from ball milling and hot pressing process.
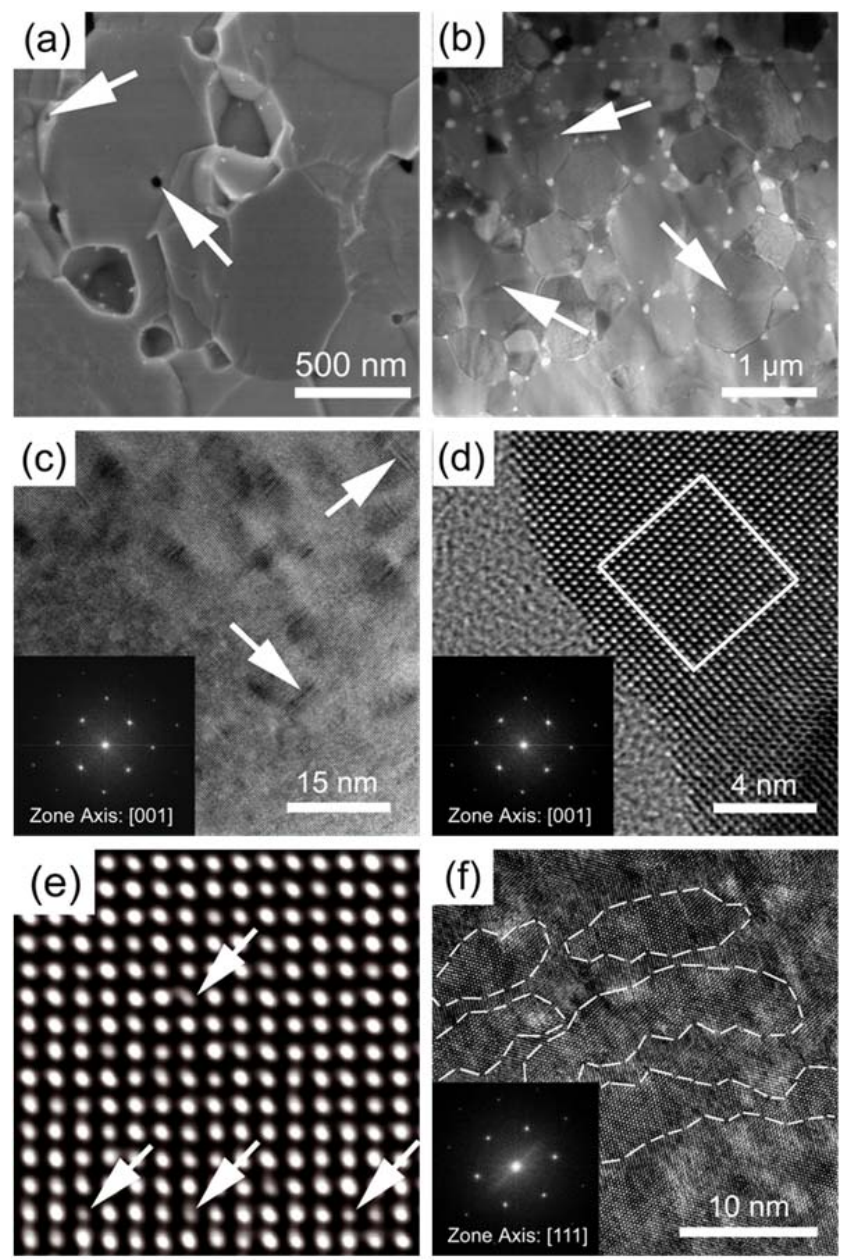

Fig. 5 (a) SEM microphotograph of freshly fractured surface, (b) bright field TEM image of typical PbSe:Al microstructure, (c) HRTEM image of the $\mathrm{Pb}$-depleted disks lying on the $\{100\}$ crystal planes, (d) atomic scaled lattice image showing the edge of the crystal foil in (c), (e) enlarged and modified HRTEM image from (d), and (f) nanoscaled subgrains and the subgrain boundaries.

\section{Conclusions}

In summary, with the enhancement of Seebeck coefficient due to the increase of local DOS near Fermi level by Al created resonant states, and the reduction of the lattice thermal conductivity by numerous distinctive microstructures, such as $\mathrm{Pb}$ depleted discs, small grains, and $\sim 10 \mathrm{~nm}$ subgrains, we report an enhanced $Z T \sim 1.3$ at $850 \mathrm{~K}$, which is $\sim 300 \%$ and $\sim 40 \%$ higher than the undoped and $\mathrm{Cl}$-doped $\mathrm{PbSe}$ samples, respectively. By accurately controlling the alignment between Fermi level and resonant states through other more appropriate dopants, further improvement on power factor and $Z T$ could be expected.

\section{Acknowledgements}

The work is partly funded by the US Department of Energy under contract number DOE DE-FG02-08ER46516 (QYZ and ZFR) and partly by the "Solid State Solar-Thermal Energy Conversion Center (S TEC)", an Energy Frontier Research Center founded by the U.S. Department of Energy, Office of Science, Office of Basic Energy Science under award number DE-SC0001299/DEFG02-09ER46577 (GC and ZFR). 


\section{References}

1. G. J. Snyder and E. S. Toberer, Nature Materials, 2008, 7, 105-114.

2. G. Chen, M. S. Dresselhaus, G. Dresselhaus, J. P. Fleurial and T. Caillat, International Materials Reviews, 2003, 48, 45-66.

3. B. Poudel, Q. Hao, Y. Ma, Y. C. Lan, A. Minnich, B. Yu, X. A. Yan, D. Z. Wang, A. Muto, D. Vashaee, X. Y. Chen, J. M. Liu, M. S. Dresselhaus, G. Chen and Z. F. Ren, Science, 2008, 320, 634-638.

4. J. P. Heremans, V. Jovovic, E. S. Toberer, A. Saramat, K. Kurosaki, A. Charoenphakdee, S. Yamanaka and G. J. Snyder, Science, 2008, 321, 554-557.

5. Y. Pei, X. Shi, A. LaLonde, H. Wang, L. Chen and J. Snyder, Nature, 2011, 473, 66-69.

6. J. P. Heremans, C. M. Thrush and D. T. Morelli, Physical Review B, 2004, 70, 115334.

7. J. Martin, L. Wang, L. Chen and G. S. Nolas, Physical Review B, 2009, 79, 115311.

8. S. V. Faleev and F. Leonard, Physical Review B, 2008, 77, 214304.

9. W. S. Liu, L. D. Zhao, B. P. Zhang, H. L. Zhang and J. F. Li, Applied Physics Letters, 2008, 93, 042109.

10. V. I. Kaidanov, S. A. Nemov and Y. I. Ravich, Soviet Physics Semiconductors-Ussr, 1992, 26, 113-125.

11. M. Zebarjadi, G. Joshi, G. H. Zhu, B. Yu, A. Minnich, Y. C. Lan, X. W. Wang, M. Dresselhaus, Z. F. Ren and G. Chen, Nano Letters, $2011, \mathbf{1 1}, 2225-2230$

12. K. Biswas, J. Q. He, Q. C. Zhang, G. Y. Wang, C. Uher, V. P. Dravid and M. G. Kanatzidis, Nature Chemistry, 2011, 3, 160-166.

13. P. F. R. Poudeu, J. D'Angelo, A. D. Downey, J. L. Short, T. P. Hogan and M. G. Kanatzidis, Angewandte Chemie-International Edition, 2006, 45, 3835-3839.

14. K. F. Hsu, S. Loo, F. Guo, W. Chen, J. S. Dyck, C. Uher, T. Hogan, E. K. Polychroniadis and M. G. Kanatzidis, Science, 2004, 303, 818-821.

15. J. Androulakis, C. H. Lin, H. J. Kong, C. Uher, C. I. Wu, T. Hogan, B. A. Cook, T. Caillat, K. M. Paraskevopoulos and M. G. Kanatzidis, J Am Chem Soc, 2007, 129, 9780-9788.

16. D. Parker and D. J. Singh, Physical Review B, 2010, 82, 035204.

17. H. Wang, Y. Z. Pei, A. D. LaLonde and G. J. Snyder, Advanced Materials, 2011, 23, 1366-1370.

18. G. T. Alekseeva, E. A. Gurieva, P. P. Konstantinov, L. V. Prokofeva and M. I. Fedorov, Semiconductors, 1996, 30, 1125-1127.

19. J. Androulakis, Y. Lee, I. Todorov, D. Y. Chung and M. Kanatzidis, Physical Review B, 2011, 83, 195209.

20. A. D. LaLonde, Y. Pei and G. J. Snyder, Energy \& Environmental Science, 2011, 6, 2090-2096.

21. X. Yan, G. Joshi, W. S. Liu, Y. C. Lan, H. Wang, S. Y. Lee, J. W. Simonson, S. J. Poon, T. M. Tritt, G. Chen and Z. F. Ren, Nano Letters, 2008, 11, 556-560.

22. B. Yu, Q. Y. Zhang, H. Wang, X. W. Wang, H. Z. Wang, D. Z. Wang, H. Z. Wang, G. J. Snyder, G. Chen and Z. F. Ren, Journal of Applied Physics, 2010, 108, 016104.

23. L. V. Prokofeva, E. A. Gurieva, S. M. Zhumaksanov, P. P. Konstantinov, K. R. Mailina, Y. I. Ravich and L. S. Stilbans, Soviet Physics Semiconductors-Ussr, 1987, 21, 1078-1080.

24. W. S. Liu, Q. Y. Zhang, Y. C. Lan, S. Chen, X. Yan, Q. Zhang, H. Wang, D. Z. Wang, G. Chen, Z. F. Ren, Adv. Energy Mater., 2011, 1, 577-587.

25. A. F. May, E. S. Toberer, A. Saramat and G. J. Snyder, Physical Review B, 2009, 80, 125205.

26. K. Xiong, G. Lee, R. P. Gupta, W. Wang, B. E. Gnade, K. Cho, Journal of Physics D, 2010, 43, 4050403.

27. A. N. Veis and Y. V. Glebova, Izvestiya Vysshikh Uchebnykh Zavedenii Fizika, 1983, 26, 117-119.

28. A. Popescu, L. M. Woods, Appllied Physics Letters, 2010, 97, 052102.

29. Z. Y. Fan, H. Q. Wang, J. C. Zheng, Journal of Applied Physics, 2011, 109, 073717.

30. Y. Zhang, X. Z. Ke, C. F. Chen, J. H. Yang, P. R. C. Kent, Physic Review Letters, 2011, 106, 206601.

31. W. S. Liu, B. P. Zhang, J. F. Li, H. L. Zhang, L. D. Zhao, Journal of Applied Physics, 2007, 102, 103717.

32. M. Brieseck, W. Lengauer, B. Gneiss, K. Wagner and S. Wagner, Microchimica Acta, 2010, 168, 309-316.

33. H. Z. Wang, Q. Zhang, B. Yu, H. Wang, W. Liu, G. Chen and Z. Ren, Journal of Materials Research, 2011 26, $912-916$. 